\title{
Effect of Chemical Reaction and Heat Absorption on MHD Nanoliquid Flow Past a Stretching Sheet in the Presence of a Transverse Magnetic Field
}

\author{
Raj Nandkeolyar ${ }^{1, *(D)}$, Bhupesh Kumar Mahatha ${ }^{2}$, Goutam Kumar Mahato ${ }^{3}$ \\ and Precious Sibanda 4 \\ 1 School of Mathematics, Thapar Institute of Engineering \& Technology, Patiala-147004, India \\ 2 Department of Mathematics, School of Applied Sciences, Kalinga Institute of Industrial Technology, \\ Bhubaneswar-751024, India; bhupeshmahatha@gmail.com \\ 3 Department of Mathematics, Centurion University of Technology \& Management, \\ Bhubaneswar-752050, India; mahatogk@gmail.com \\ 4 School of Mathematics, Statistics \& Computer Science, University of KwaZulu-Natal, Private Bag X01, \\ Scottsville 3209, Pietermaritzburg, South Africa; sibandap@ukzn.ac.za \\ * Correspondence: rajnandkeolyar@gmail.com; Tel.: +91-7837-1813-19
}

Received: 24 September 2017; Accepted: 24 February 2018; Published: 27 February 2018

\begin{abstract}
In this paper, authors investigate homogeneous-heterogeneous chemical reaction and heat absorption effects on a two-dimensional steady hydromagnetic Newtonian nanoliquid flow along a continuously stretching sheet. The flow field is subjected to a uniform magnetic field acting in a direction perpendicular to the direction of nanoliquid flow. A mathematical model of the physical problem is presented involving nonlinear partial differential equations with appropriate boundary conditions. These equations are then transformed into nonlinear ordinary differential equations using a suitable similarity transformation. Finally, approximate solutions of the transformed equations are obtained using the spectral quasi-linearization method. Results of fluid velocity, fluid temperature, and species concentration are depicted graphically, while the values of skin friction and Nusselt number are presented in tabular form. Fluid flow models of this kind find applications in catalytic reactors involving chemical reactions, insulation systems, and in heat exchangers. The applied magnetic field has a retarding influence on the nanofluid velocity and species concentration, while it does not have any significant effect on the nanofluid temperature. The homogeneous and heterogeneous reactions tend to decrease the species concentration.
\end{abstract}

Keywords: homogeneous-heterogeneous reactions; heat absorption; hydromagnetic flow; stretching sheet; SQLM

\section{Introduction}

Nanofluids have many applications in industries, such as, in cooling of electronic circuits and in enhancing heat transfer characteristics in geothermal heat source pumps. These are conventional base fluids containing 1-50 nm sized nanoparticles of metals and oxides. The enhanced thermal properties of nanofluids were first reported by Choi et al. [1]. Choi et al. [1] observed that the thermal conductivity of the base fluids can be enhanced significantly by mixing a mere amount (about 0.01 fraction by volume) of nanoparticles in the base fluid. Consequently, studies involving the flow of nanofluids were investigated by several researchers [2-5] to examine the different aspects of the problem. The effect of magnetic field on the flow of a viscous and electrically conducting fluid is known to be significant and is crucial in industrial and engineering applications, such as, in magnetohydrodynamic (MHD) pumps, generators, accelerators, and flow meters. In spite of its important applications in industry, there are 
very few research studies that highlight the consequences of an externally applied transversal magnetic field on the flow and heat transfer characteristics along a stretching/shrinking sheet. An investigation on the influence of magnetic field on a transient nanofluid flow including the melting and heat sink influences was presented by Chamkha et al [6]. They concluded that an increase in Hartmann number tends to increase the coefficient of skin friction. Ibrahim et al. [7] analyzed the two-dimensional MHD nanofluid flow near a stagnation point including the effects of Brownian motion and thermophoresis diffusion. They found that the momentum boundary layer gets thinner with increasing values of the magnetic parameter.

There are several fluid engineering devices where the temperature difference between the boundary layer fluid and surface plays an important role. Heat generation/absorption effects produced due to temperature-differences have significant implications on heat transfer characteristics, such as, in processes where the working fluid undergoes exothermic/endothermic chemical reactions [8], and in preparation of metal waste obtained as a by-product from used nuclear fuel [9]. Thus, the studies involving heat sources/sinks have become a central point of attraction for the researchers interested in investigating fluid dynamic problems. The effect of heat generation/absorption on the hydromagnetic flow and heat transfer along a semi-infinite flat plate in a saturated porous medium was examined by Chamkha and Khaled [10]. They considered two different cases viz. uniform heat flux and uniform wall temperature at the plate. The similarity solution for steady state problem of free convection hydromagnetic liquid flow from an oblique plate was obtained by Chamkha and Khaled [10]. The transient convective heat and mass transfer flow towards a vertical surface under the influence of an external magnetic field and heat generation/absorption was analytically investigated by Kamel [11]. The unsteady two-dimensional magnetohydrodynamic flow of a heat absorbing fluid considering the effects of thermal and mass buoyancy was examined by Chamkha [12]. Alam et al. [13] presented a numerical investigation for the simultaneous free and forced convection flow over a permeable surface with an aim to highlight the impact of heat generation. Alam et al. [14] later investigated the combined influences of chemical reaction and heat generation on the hydromagnetic flow over a permeable surface. Recently, Mahatha et al. [15] investigated the non-uniform heat generation/asborption effects on the hydromagnetic stagnation point boundary layer flow of a nanofluid over a stretching sheet with Newtonian heating at the surface.

Systems involving chemical reactions are complicated. Chaudhary and Merkin [16,17] examined the influences of both homogeneous and heterogeneous reactions on the stagnation point viscous fluid flow. They presented two simple models for both the reactions in a boundary layer stagnation-point flow considering (i) equal and (ii) different diffusivities for reactant and auto-catalyst. Merkin [18] studied the viscous flow along a flat plate in the presence of both the reactions. This study was later extended by Chaudhary and Merkin [19] to include the effect of loss of auto-catalyst. They obtained numerical solutions near the leading edge of a plate. Khan and Pop [20] examined the stagnation point flow on a porous wall considering the effects of both the chemical reactions. Khan and Pop [21] also investigated the influences of homogeneous and heterogeneous reactions on a viscoelastic fluid flow along a stretching surface. They encountered a reducing effect of the viscoelastic parameter on the species concentration at the surface. Recently, Kameswaran et al. [22,23] and Nandkeolyar et al. [24] analyzed the influences of both these chemical reactions on a nanofluid flow considering different aspects of the problem.

Motivated by aforementioned research studies, our aim is to analyze the effects of homogeneous and heterogeneous chemical reactions due to foreign species and heat absorption on the two-dimensional laminar boundary layer flow of a Newtonian nanofluid along a stretching surface under the influence of an externally applied uniform transverse magnetic field. The heat absorption phenomena play an important role in many fluid engineering devices and affect the heat transfer characteristics in the flow-field. There is no study in the literature that provides an insight into the combined influences of heat absorption, heterogeneous and homogeneous chemical reactions on the magnetohydrodynamic flow of a Newtonian nanofluid under the conditions of the present problem. 


\section{The Mathematical Formulation}

Let us consider the steady fully developed hydromagnetic flow of a heat absorbing $\mathrm{Cu}$-water nanoliquid along a continuously stretching sheet in the presence of two chemically reacting foreign species $A$ and $B$ with concentrations $a$ and $b$, respectively. The length of the sheet is taken along the $x$ axes whereas the $y$ axis is taken normal to the sheet. The thermophysical properties of $C u$ nanoparticles and pure water are presented in Table 1 . The fluid flow, in the region $y>0$, is caused by linear stretching of the sheet with velocity $U_{w}(x)$ along its length keeping the position of the origin $O$ fixed. The fluid flow is permeated by a uniform magnetic field of strength $B_{0}$ acting along $y$-axis. The fluid temperature at the surface is maintained at $T_{w}(x)$ whereas outside the boundary layer region, it is at a constant temperature $T_{\infty}$. The flow configuration and axes system are depicted in Figure 1 . It is further assumed that

- the flow is not subjected to polarized/applied voltages so that the effect of polarization of voltages is neglected [25],

- the magnetic Reynolds number of the fluid is very small so that the induced magnetic field effects are neglected as compared to the applied magnetic field,

- the effect of buoyancy forces are ignored,

- the base fluid and nanoparticles are in thermal equilibrium and there is no slip between them,

- it is assumed that a homogeneous reaction takes place between the species $A$ and $B$ as [16]

$$
A+2 B \rightarrow 3 B, \quad \text { rate }=k_{c} a b^{2}
$$

while a heterogeneous first order, isothermal reaction undergoes on the catalyst surface, as

$$
A \rightarrow B, \quad \text { rate }=h_{s} a
$$

where $k_{c}$ and $h_{s}$ are homogeneous and heterogeneous reaction rates, respectively. It is further assumed that no energy is released during these reactions.

$$
u \rightarrow 0, v \rightarrow 0, T \rightarrow T_{\infty}, a \rightarrow a_{0}, b \rightarrow 0 \text { as } y \rightarrow \infty
$$

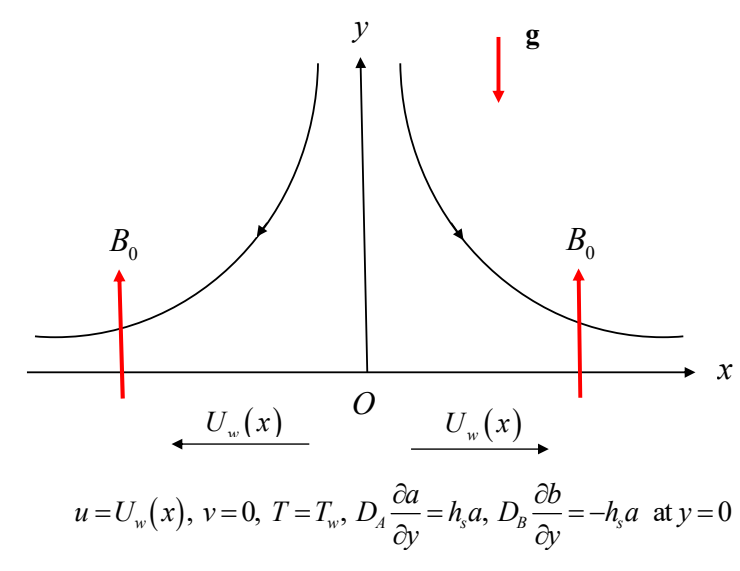

Figure 1. Flow configuration and boundary conditions.

Table 1. Physical properties of water and $C u$ particles.

\begin{tabular}{lccc}
\hline Nanofluid Component & $\rho\left(\mathbf{k g} / \mathbf{m}^{\mathbf{3}}\right)$ & $\mathrm{C}_{\mathrm{p}}(\mathrm{J} / \mathbf{k g K})$ & $\mathbf{k}(\mathrm{W} / \mathbf{m K})$ \\
\hline Pure water & 997.1 & 4179 & 0.613 \\
Copper $(\mathrm{Cu})$ & 8933 & 385 & 401 \\
\hline
\end{tabular}


Under the above assumptions, equations governing the conservation of mass, momentum, energy, and species concentrations, are given by

$$
\begin{aligned}
\frac{\partial u}{\partial x}+\frac{\partial v}{\partial y} & =0 \\
u \frac{\partial u}{\partial x}+v \frac{\partial u}{\partial y} & =\frac{\mu_{n f}}{\rho_{n f}} \frac{\partial^{2} u}{\partial y^{2}}-\frac{\sigma B_{0}^{2}}{\rho_{n f}} u \\
u \frac{\partial T}{\partial x}+v \frac{\partial T}{\partial y} & =\alpha_{n f} \frac{\partial^{2} T}{\partial y^{2}}-\frac{Q_{0}}{\left(\rho c_{p}\right)_{n f}}\left(T-T_{\infty}\right) \\
u \frac{\partial a}{\partial x}+v \frac{\partial a}{\partial y} & =D_{A} \frac{\partial^{2} a}{\partial y^{2}}-k_{c} a b^{2} \\
u \frac{\partial b}{\partial x}+v \frac{\partial b}{\partial y} & =D_{B} \frac{\partial^{2} b}{\partial y^{2}}+k_{c} a b^{2}
\end{aligned}
$$

where $u$ and $v$ are components of nanofluid velocity in $x$ and $y$ directions, respectively, $T$ denotes temperature of the nanofluid within the boundary layer, $\mu_{n f}, \sigma, \rho_{n f}, \alpha_{n f}$, and $Q_{0}$, are respectively, viscosity, electrical conductivity, density, thermal diffusivity, and heat absorption. The subscript ${ }_{n f}$ stands for the physical properties of nanofluid.

The dynamic viscosity $u_{n f}$, effective density $\rho_{n f}$, thermal diffusivity $\alpha_{n f}$, thermal conductivity $k_{n f}$, and heat capacitance $\left(\rho c_{p}\right)_{n f}$ of the nanofluid are defined as [26-29]

$$
\begin{aligned}
\mu_{n f} & =\frac{\mu_{f}}{(1-\phi)^{2.5}}, \\
\rho_{n f} & =(1-\phi) \rho_{f}+\phi \rho_{s}, \\
\alpha_{n f} & =\frac{k_{n f}}{\left(\rho c_{p}\right)_{n f}}, \\
\frac{k_{n f}}{k_{f}} & =\frac{k_{s}+2 k_{f}-2 \phi\left(k_{f}-k_{s}\right)}{k_{s}+2 k_{f}+2 \phi\left(k_{f}-k_{s}\right)}, \\
\left(\rho c_{p}\right)_{n f} & =(1-\phi)\left(\rho c_{p}\right)_{f}+\phi\left(\rho c_{p}\right)_{s} .
\end{aligned}
$$

The subscripts ${ }_{f}$ and ${ }_{s}$, in the above equations, are used to denote the physical properties of the base fluid and nanoparticles, respectively, and $\phi$ denotes the volume fraction of the nanoparticles.

The appropriate boundary conditions for the flow problem are

$$
\begin{gathered}
\text { At } y=0: u=U_{w}(x)=\frac{c x}{L}, v=0, T=T_{w}=T_{\infty}+d\left(\frac{x}{L}\right)^{2}, D_{A} \frac{\partial a}{\partial y}=h_{s} a, D_{B} \frac{\partial b}{\partial y}=-h_{s} a ; \\
\text { As } y \rightarrow \infty: u \rightarrow 0, T \rightarrow T_{\infty}, a \rightarrow a_{0}, b \rightarrow 0 .
\end{gathered}
$$

where $c$ is a constant which measures the stretching rate of the sheet, $d$ is a proportionality constant used for measuring a rise in the boundary layer temperature with increasing $x, a_{0}$ denotes the concentration of the foreign species $A$ outside the boundary layer. The symbol $L$ denotes the characteristic length of the flow which, in this case, may be defined as the thickness of the boundary layer. We now chose a transformation given by

$$
\psi(x, y)=\sqrt{\frac{c v_{f}}{L}} x f(\eta), \theta=\frac{\left(T-T_{\infty}\right)}{\left(T_{w}-T_{\infty}\right)}, a=a_{0} g(\eta), b=a_{0} h(\eta), \eta=\sqrt{\frac{c}{v_{f} L}} y,
$$


so that the conservation of mass Equation (3) is satisfied, and Equations (4)-(7) are transformed to a set of similarity equations, given by

$$
\begin{aligned}
& f^{\prime \prime \prime}+\phi_{1} f f^{\prime \prime}-\phi_{1} f^{\prime 2}-M \phi_{2} f^{\prime}=0, \\
& \theta^{\prime \prime}+\operatorname{Pr} \phi_{3} f \theta^{\prime}-2 \operatorname{Pr} \phi_{3} f^{\prime} \theta-\operatorname{Pr} \beta_{h} \phi_{4} \theta=0, \\
& g^{\prime \prime}+S c f g^{\prime}-S c K g h^{2}=0, \\
& h^{\prime \prime}+\frac{S c}{\delta} f h^{\prime}+\frac{S c}{\delta} K g h^{2}=0,
\end{aligned}
$$

where

$$
\begin{aligned}
& M=\frac{\sigma B_{0}^{2} L}{\rho_{f} c}, P r=\frac{v_{f}}{\alpha_{f}}, \beta_{h}=\frac{Q_{0} L}{c\left(\rho c_{p}\right)_{f}}, S c=\frac{v_{f}}{D_{A}}, K=\frac{k_{c} a_{0}^{2} L}{c}, \phi_{1}=(1-\phi)^{2.5}\left\{(1-\phi)+\phi\left(\frac{\rho_{s}}{\rho_{f}}\right)\right\}, \\
& \phi_{2}=(1-\phi)^{2.5}, \phi_{3}=\frac{\left\{k_{s}+2 k_{f}+2 \phi\left(k_{f}-k_{s}\right)\right\}\left\{1-\phi+\phi \frac{\left(\rho c_{p}\right)_{s}}{\left(\rho c_{p}\right)_{f}}\right\}}{\left\{k_{s}+2 k_{f}-2 \phi\left(k_{f}-k_{s}\right)\right\}}, \phi_{4}=\frac{k_{s}+2 k_{f}+2 \phi\left(k_{f}-k_{s}\right)}{k_{s}+2 k_{f}-2 \phi\left(k_{f}-k_{s}\right)}, \\
& \text { and } \delta=\frac{D_{B}}{D_{A}} .
\end{aligned}
$$

The non-dimensional parameters defined above are, Prandtl number $\mathrm{Pr}$, magnetic parameter $M$, Schmidt number $S c$, heat absorption parameter $\beta_{h}$, homogeneous reaction rate $K$, and the ratio of mass diffusion constants $\delta$. The functions $\phi_{1}, \phi_{2}, \phi_{3}$ and $\phi_{4}$ depend on the thermal properties of the nanoparticles and the base fluid, and are dimensionless.

Boundary conditions (13) and (14), in non-dimensional form, are

$$
\begin{aligned}
& \text { At } \eta=0: f=0, f^{\prime}=1, \theta=1, g^{\prime}=K_{s} g, \delta h^{\prime}=-K_{s} g ; \\
& \text { As } \eta \rightarrow \infty: f^{\prime} \rightarrow 0, \theta \rightarrow 0, g \rightarrow 1, h \rightarrow 0,
\end{aligned}
$$

where $K_{s}=\frac{h_{s}}{D_{A}} \sqrt{\frac{v_{f} L}{c}}$ measures the heterogeneous reaction's strength.

Assuming that the chemical species $A$ and $B$ have equal diffusivities i.e., $\delta=1$ [16], we obtain,

$$
g(\eta)+h(\eta)=1
$$

Equations (18) and (19) under this assumption, reduce to

$$
g^{\prime \prime}+S c f g^{\prime}-S c K g(1-g)^{2}=0,
$$

subject to the conditions

$$
g^{\prime}(n)=K_{s} g(n) \text { at } \eta=0 \text {, and } g(\eta) \rightarrow 1 \text { as } \eta \rightarrow \infty .
$$

Thus the problem is now described by the Equations (16), (17), and (23) with the boundary conditions (20), (21) and (24). One may also wish to obtain the values of local skin friction coefficient $C_{f_{x}}$ and local Nusselt number $N u_{x}$, which for the present problem are

$$
\begin{aligned}
& C_{f_{x}} R e_{x}^{1 / 2}=\frac{1}{(1-\phi)^{2.5}} f^{\prime \prime}(0), \\
& N u_{x} / R e_{x}^{1 / 2}=-\frac{1}{\phi_{4}} \theta^{\prime}(0) .
\end{aligned}
$$

where $R e_{x}=\frac{x U_{w}(x)}{v}$ is local Reynolds number. 


\section{Solution Procedure}

The numerical solution of the mathematical model represented by Equations (16), (17), and (23) together with the boundary conditions (20), (21), and (23), respectively, is obtained using a quasi-linearization technique known as Spectral quasi-linearization method (SQLM) which is successfully employed for solving fluid flow problems by Motsa et al. [30]. In view of the framework of SQLM, the iteration scheme is obtained as

$$
\begin{aligned}
& f_{r+1}^{\prime \prime \prime}+a_{1, r} f_{r+1}^{\prime \prime}+a_{2, r} f_{r+1}^{\prime}+a_{3, r} f_{r+1}=-a_{4, r}, \\
& b_{1, r} f_{r+1}^{\prime}+b_{2, r} f_{r+1}+\theta_{r+1}^{\prime \prime}+b_{3, r} \theta_{r+1}^{\prime}+b_{4, r} \theta_{r+1}=-b_{5, r}, \\
& c_{1, r} f_{r+1}+g_{r+1}^{\prime \prime}+c_{2, r} g_{r+1}^{\prime}+c_{3, r} g_{r+1}=-c_{4, r},
\end{aligned}
$$

where

$$
\begin{aligned}
& a_{1, r}=\phi_{1} f_{r}, a_{2, r}=-2 \phi_{1} f_{r}^{\prime}-M \phi_{2}, a_{3, r}=\phi_{1} f_{r}^{\prime \prime}, \quad a_{4, r}=-\phi_{1}\left(f_{r} f_{r}^{\prime \prime}-f_{r}^{\prime 2}\right), \\
& b_{1, r}=-2 \operatorname{Pr} \phi_{3} \theta_{r}, \quad b_{2, r}=\operatorname{Pr} \phi_{3} \theta_{r}^{\prime}, \quad b_{3, r}=\operatorname{Pr} \phi_{3} f_{r}, \quad b_{4, r}=-\operatorname{Pr}\left(2 \phi_{3} f_{r}^{\prime}+\beta_{h} \phi_{4}\right), \\
& b_{5, r}=\operatorname{Pr}\left(\phi_{3} f_{r} \theta_{r}^{\prime}-2 \phi_{3} f_{r}^{\prime} \theta_{r}\right), c_{1, r}=S c g_{r}^{\prime}, c_{2, r}=\operatorname{Sc} f_{r}, c_{3, r}=\operatorname{ScK}\left(-1+4 g_{r}-3 g_{r}^{2}\right), \\
& c_{4, r}=\operatorname{Sc}\left(-f_{r} g_{r}^{\prime}-2 K g_{r}^{2}+2 K g_{r}^{3}\right) .
\end{aligned}
$$

The boundary conditions for the above iteration scheme are

$$
\begin{aligned}
& f_{r+1}(0)=0, \quad f_{r+1}^{\prime}(0)=1, \quad f_{r+1}^{\prime}(\infty) \rightarrow 0, \\
& \theta_{r+1}(0)=1, \quad \theta_{r+1}(\infty) \rightarrow 0, \\
& g_{r+1}^{\prime}(0)=K_{s} g_{r+1}(0), \quad g_{r+1}(\infty) \rightarrow 1,
\end{aligned}
$$

The linearized Equations (27)-(29) are then treated with the Chebyshev pseudo-spectral collocation method whose details are well presented by Motsa et al. [30] and Nandkeolyar et al. [24]. The method requires initial guesses to start with, which are taken as

$$
f_{0}(\eta)=1-e^{-\eta}, \theta_{0}(\eta)=e^{-\eta}, g_{0}(\eta)=1-0.5 e^{-K_{s} \eta} .
$$

In order to validate the results obtained by the spectral quasilinearization method, an error analysis has been carried out. The infinity norm of the error in function values of two consecutive iterations is examined and is set to meet the tolerance level $\epsilon$. At the $(r+1)^{\text {th }}$ level of iteration, the maximum error $E_{d}$ is defined as

$$
E_{d}=\max \left(\left\|z_{1, r+1}-z_{1, r}\right\|_{\infty},\left\|z_{2, r+1}-z_{2, r}\right\|_{\infty}, \ldots,\left\|z_{m, r+1}-z_{m, r}\right\|_{\infty}\right)
$$

where $z_{i} ; i=1,2, \ldots, m$ are the unknown functions in the nonlinear system. The values of $E_{d}$ for different iteration levels are plotted in Figure 2. For the computations presented in the paper $\epsilon=10^{-12}$ and it can be seen that about 60 iterations are adequate for the SQLM iteration scheme to suffice the prescribed tolerance limit $\epsilon$. This accuracy level in $f^{\prime}$ and $g$ are attained in a mere seven iterations. An inspection analysis is carried out to investigate the required number of collocation points $N$ and the value of the variable $L$ which is used to approximate $\infty$. At first, a suitable value of $L_{\infty}$ is determined by successively increasing its value so that no further changes in the results are observed and that the far boundary conditions are satisfied. For the present problem, a suitable value of $L_{\infty}$ is found to be $L_{\infty}=20$. The far conditions are satisfied even for smaller values but it is only when we take $L_{\infty}=20$ that the results do not vary any further. The same steps are repeated for finding an appropriate value of $N$ and it is found that $N=80$ is sufficient to achieve the prescribed accuracy level. 


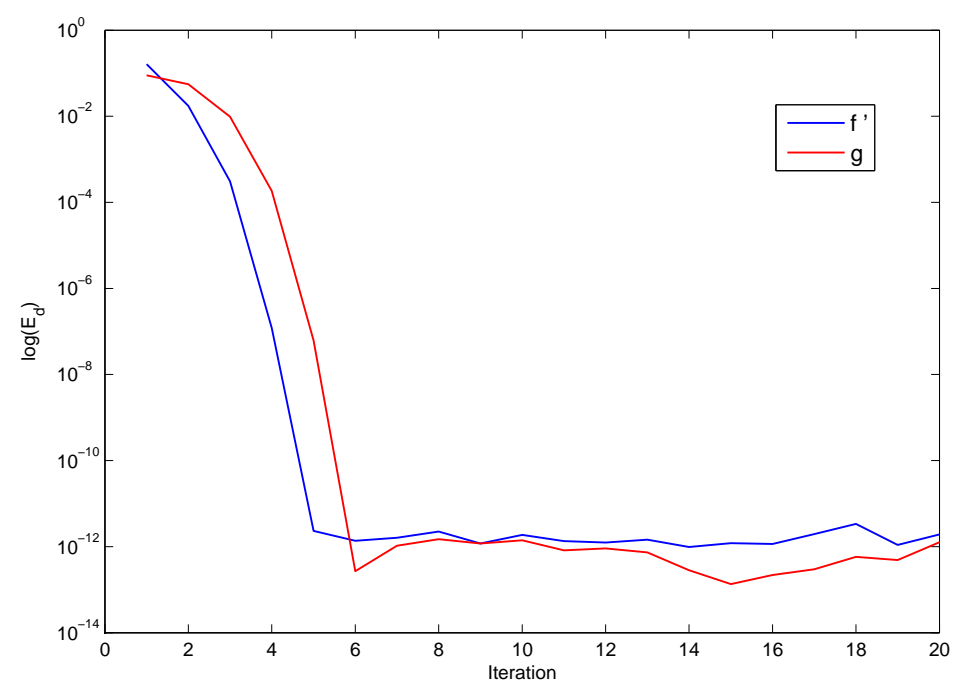

(a)

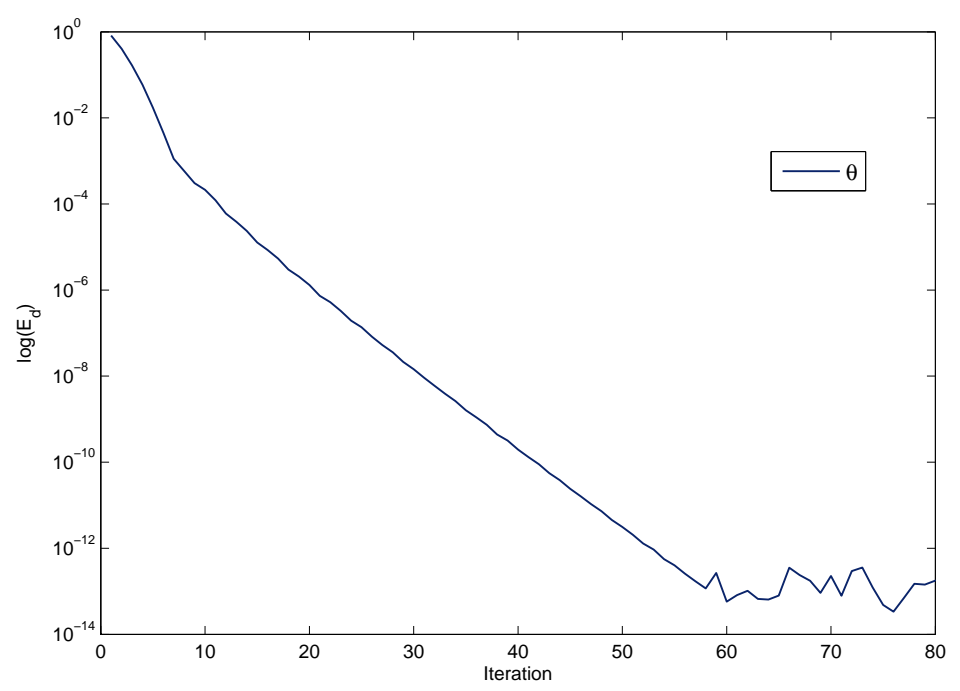

(b)

Figure 2. Maximum error in (a) $f^{\prime}$ and $g$, (b) $\theta$ versus number of iterations.

\section{Results and Discussion}

In order to analyze the effects of nanoparticle volume fraction and magnetic field on the fully developed flow-field, the profiles of fluid velocity $f^{\prime}(\eta)$, fluid temperature $\theta(\eta)$, and species concentration $g(\eta)$, for several values of nanoparticle volume fraction $\phi$ and magnetic parameter $M$, within the boundary layer region, are depicted in Figure 3 and 4 taking $S c=1$ and $P r=6.7850$. The effects of homogeneous and heterogeneous reactions on species concentration and heat absorption on fluid temperature are shown in Figure 5. In Table 2, we display the variations in $C_{f_{x}}$ and $N u_{x}$ with respect to the pertinent flow parameters.

The amount of $\mathrm{Cu}$ nanoparticles mixed in the base fluid is measured with the nanoparticle volume fraction parameter $\phi$, and the effect of this parameter on the profiles of nanofluid velocity $f^{\prime}(\eta)$, nanofluid temperature $\theta(\eta)$, and species concentration $g(\eta)$ is depicted in Figure 3. It is shown in Figure 3 that an increase in nanoparticle volume fraction increases the nanofluid velocity, species concentration, and nanofluid temperature within the boundary layer region. One must note that increasing the value of nanoparticle volume fraction beyond a critical value must start decreasing the fully developed fluid velocity. However, at least for the values of volume fraction used, the fluid 
velocity is found to be increasing with increasing nanoparticle volume fraction. All the three boundary layers, namely, the momentum boundary layer, the thermal boundary layer, and the concentration boundary layer get thicker with increasing nanoparticle volume fraction.

The application of externally applied magnetic field $B_{0}$ on the flow of a viscous, incompressible, and electrically conducting fluid gives rise to a mechanical force, known as Lorentz force and the strength of the applied magnetic field is represented by the non-dimensional magnetic parameter $M$. Figure 4 is presented to show the effect of the magnetic parameter $M$ on the nanofluid velocity, nanofluid temperature, and species concentration. It is perceived from Figure $4 a-c$ that the nanofluid velocity and species concentration both decrease whereas the nanofluid temperature increases, within the boundary layer region, with increasing magnetic parameter $M$. It is also noted that the effect of the magnetic parameter on the fluid temperature is not that significant as compared to its effect on nanofluid velocity and species concentration. Since the magnetic parameter $M$ measures the strength of the Lorentz force, we conclude that the Lorentz force has a tendency to reduce the nanofluid velocity as well as species concentration, whereas it does not have a significant effect on the nanofluid temperature. Thus, the magnetic field is found to provide a stabilization mechanism in the fluid flow which can be utilized in boundary layer flow control.

Figure $5 \mathrm{a}, \mathrm{b}$ illustrate the effects of homogeneous and heterogeneous reactions, on species concentration while the impact of heat absorption on nanofluid temperature is presented in Figure $5 c$. It is revealed in Figure $5 a, b$ that the species concentration decreases on increasing both the homogeneous reaction parameter $K$ and heterogeneous reaction parameter $K s$ within the boundary layer region. Additionally, within the boundary layer region, the nanofluid temperature also diminishes with increasing values of heat absorption parameter $\beta_{h}$. The parameters $K$ and Ks are known to represent the strength of homogeneous and heterogeneous reaction rates, respectively. Thus it can be understood that the homogeneous and heterogeneous reactions have a reducing influence on the species concentration. The heat absorption tends to reduce the fluid temperature within the boundary layer region. It is worthy to mention that the heterogeneous reaction has a stronger effect on species concentration than the homogeneous reaction.

In order to examine the effects of nanoparticle volume fraction $\phi$, magnetic field $M$, and heat absorption $\beta_{h}$ on the coefficient of skin friction and the coefficient of heat transfer at the plate, the values of the skin friction coefficient $f^{\prime \prime}(0)$ and the coefficient of heat transfer at the plate $\theta^{\prime}(0)$ are presented in Table 2 for several values of $\phi, M$, and $\beta_{h}$. In Table 2, the skin-friction coefficient $f^{\prime \prime}(0)$ and the Nusselt number $\theta^{\prime}(0)$ are found to be decreasing with increasing values of the nanoparticle volume fraction $\phi$. This decrease in skin friction and heat transfer rate is due to a respective increase in nanofluid velocity and temperature within the boundary layer region with increasing values of the nanoparticle volume fraction. This indicates that the nanoparticle volume fraction has a tendency of reducing the skin friction coefficient and the rate of heat transfer at the surface. It is witnessed from Table 2 that the heat transfer coefficient $\theta^{\prime}(0)$ is decreasing while the skin-friction coefficient $f^{\prime \prime}(0)$ is increasing with increasing values of the magnetic parameter $M$ which implies that the rate of heat transfer at the plate is reduced with increasing Lorentz force whereas it has a reverse effect on the skin friction. In addition, the heat transfer coefficient $\theta^{\prime}(0)$ is found to be an increasing function of $\beta_{h}$ which leads to the conclusion that the heat absorption tends to enhance the rate of heat transfer at the surface. 


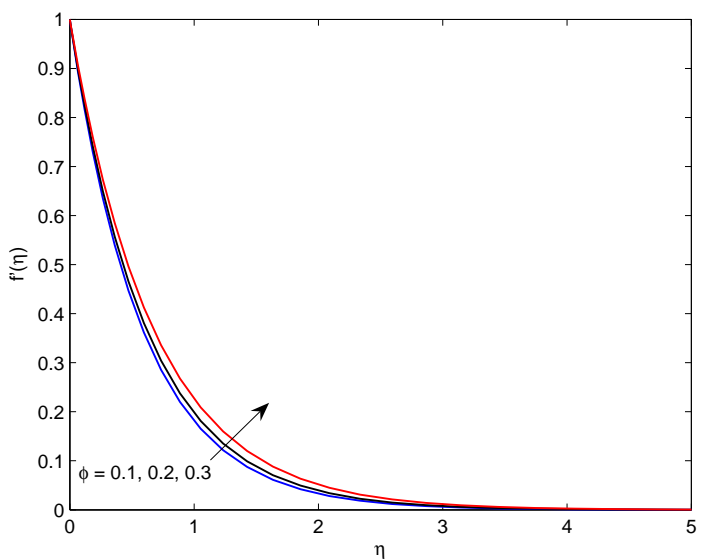

(a)

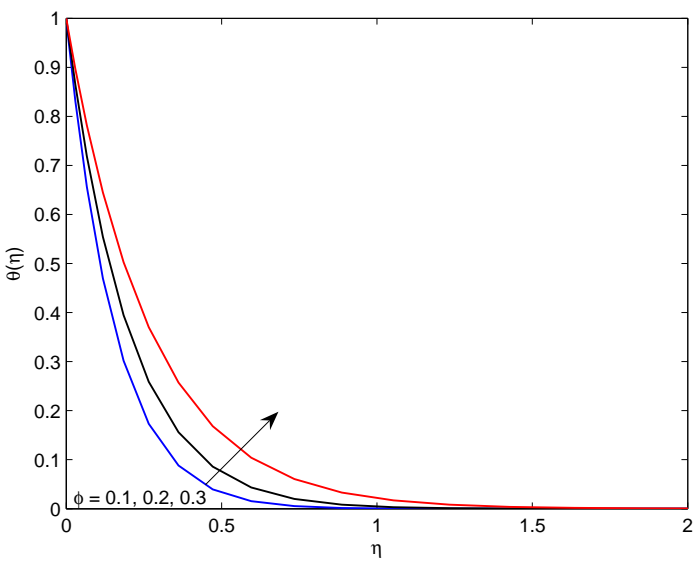

(b)

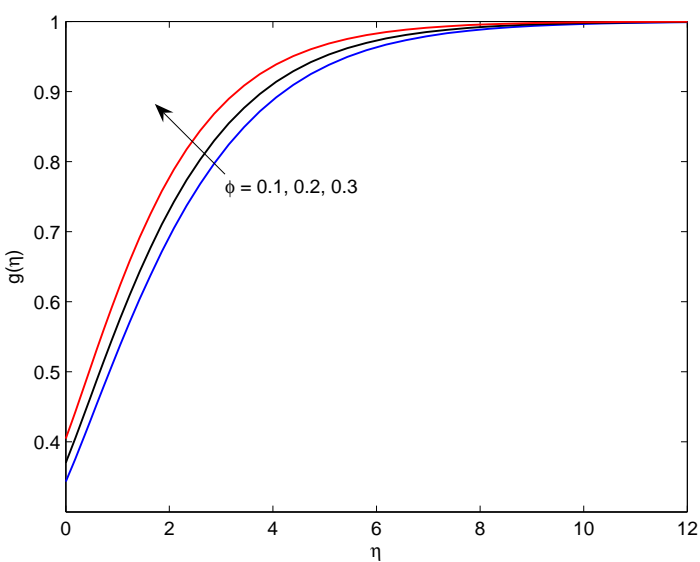

(c)

Figure 3. Variations in (a) $f^{\prime}$, (b) $\theta$, and (c) $g$ with respect to $\phi$ when $M=2, K=0.5, K_{s}=0.5, \beta_{h}=2$, $S c=1$ and $P r=6.7850$. 


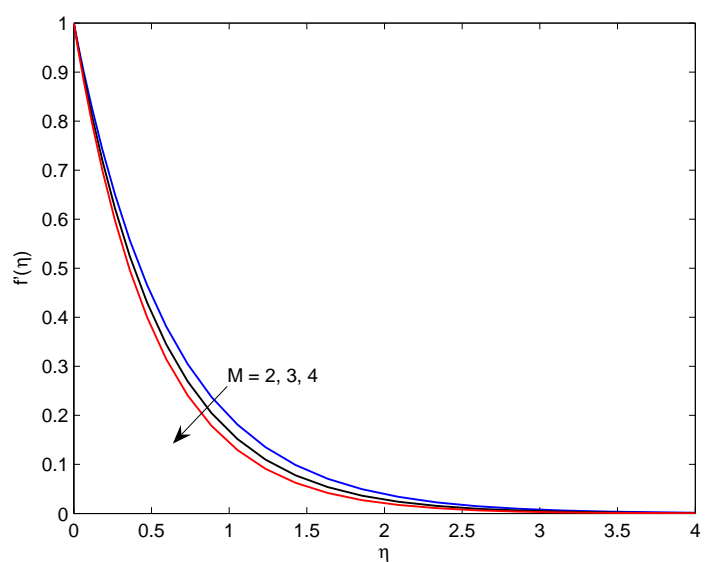

(a)

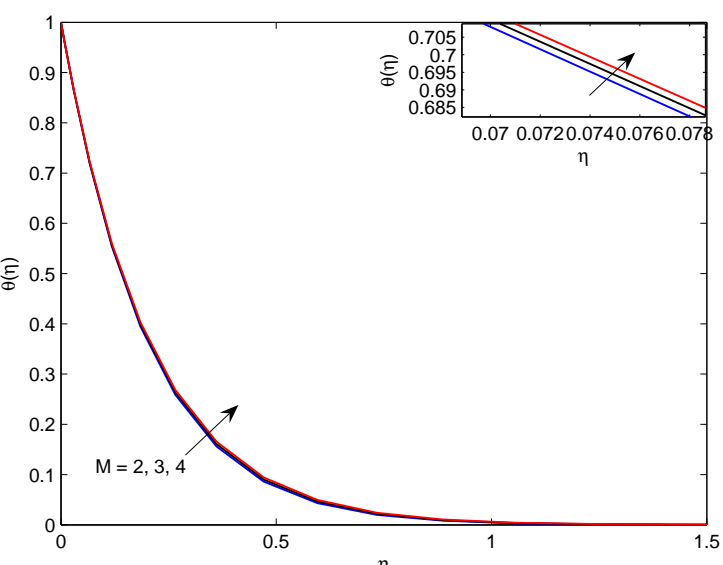

(b)

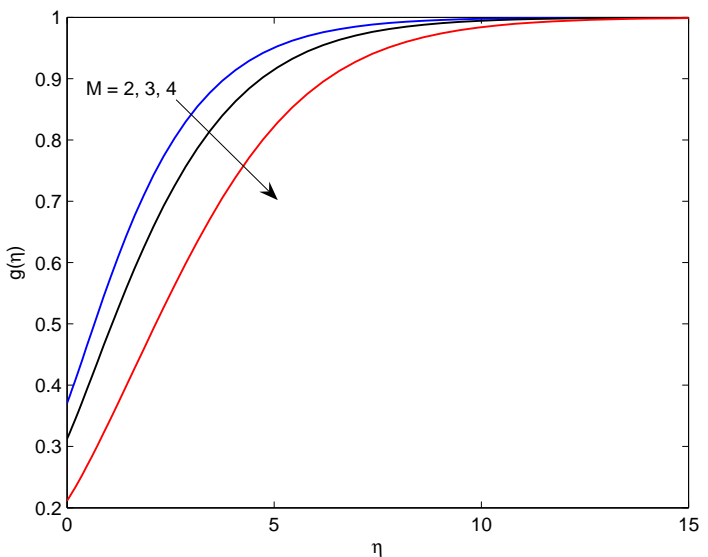

(c)

Figure 4. Variations in (a) $f^{\prime}$, (b) $\theta$, and (c) $g$ with respect to $M$ when $\phi=0.2, K=0.5, K_{s}=0.5, \beta_{h}=2$, $S c=1$, and $\operatorname{Pr}=6.7850$. 


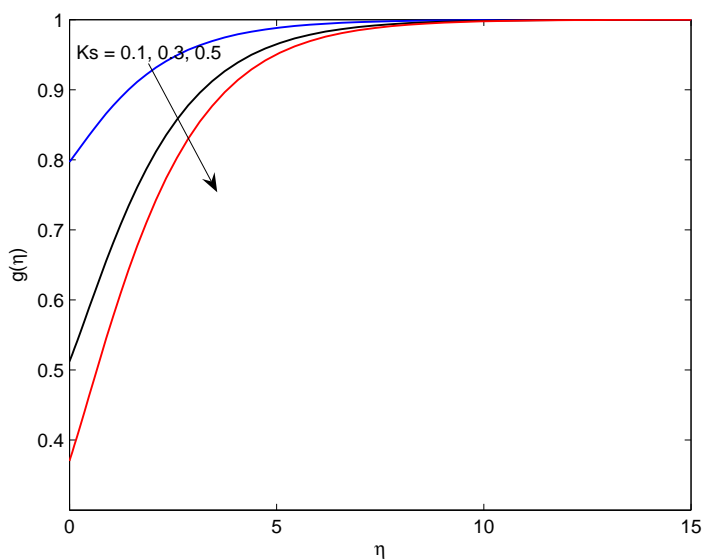

(a)

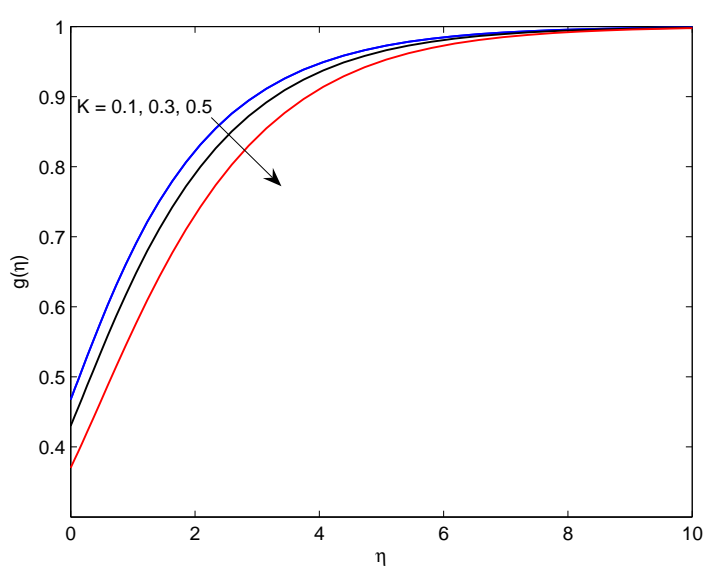

(b)

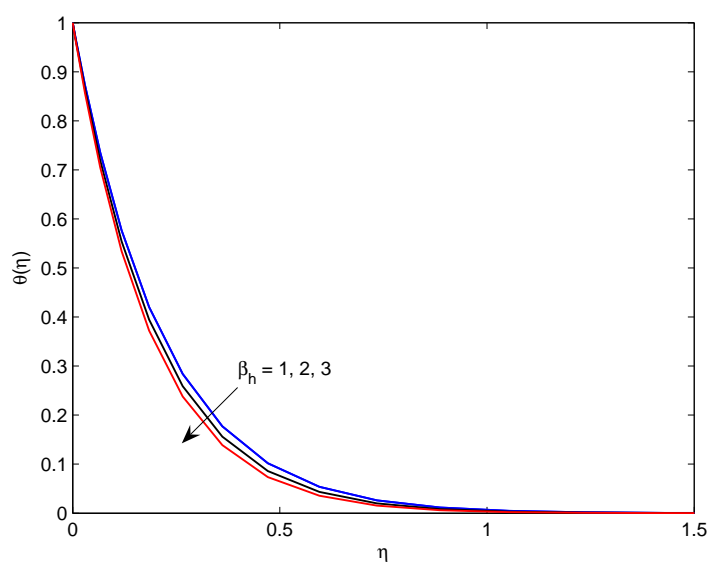

(c)

Figure 5. Variations in $g$ with respect to (a) $K_{s}$ and (b) $K$, and in (c) $\theta$ with respect to $\beta_{h}$ when $\phi=0.1$, $M=2$, and $P r=6.7850$. 
Table 2. Variations in $C_{f_{X}} R e_{x}^{1 / 2}$ and $N u_{x} R e_{x}^{-1 / 2}$ for different parameter values when $\operatorname{Pr}=6.7850$.

\begin{tabular}{ccccc}
\hline $\boldsymbol{\phi}$ & $\boldsymbol{M}$ & $\boldsymbol{\beta}_{\boldsymbol{h}}$ & $C_{f_{x}} \boldsymbol{R} \boldsymbol{e}_{x}^{\mathbf{1 / 2}}$ & $\boldsymbol{N} \boldsymbol{u}_{x} \boldsymbol{R} \boldsymbol{e}_{\boldsymbol{x}}^{-\mathbf{1 / 2}}$ \\
\hline $\mathbf{0 . 1}$ & 2 & 2 & 1.7078 & 6.2232 \\
$\mathbf{0 . 2}$ & 2 & 2 & 1.6213 & 4.9122 \\
$\mathbf{0 . 3}$ & 2 & 2 & 1.4862 & 3.6863 \\
0.2 & $\mathbf{2}$ & 2 & 1.6213 & 4.9122 \\
0.2 & $\mathbf{3}$ & 2 & 1.7891 & 4.8806 \\
0.2 & $\mathbf{4}$ & 2 & 1.9425 & 4.8518 \\
0.2 & 2 & $\mathbf{1}$ & 1.6213 & 4.5859 \\
0.2 & 2 & $\mathbf{2}$ & 1.6213 & 4.9122 \\
0.2 & $\mathbf{2}$ & $\mathbf{3}$ & 1.6213 & 5.2150 \\
\hline
\end{tabular}

\section{Conclusions}

The combined effects of homogeneous and heterogeneous chemical reactions and heat absorption on the fully developed magnetohydrodynamic Newtonian nanofluid flow past a linearly stretching sheet are investigated. The governing nonlinear ordinary differential equations are solved using a Chebyshev pseudo-spectral method based numerical technique known as spectral quasilinearization method (SQLM). The spectral quasilinearization method is found to be successful in solving the present problem. The significant findings of the study are summarized below:

1. The impact of nanoparticle volume fraction on the momentum, thermal, concentration boundary layers is to increase their thickness. The nanoparticle volume fraction has reducing influences on the skin friction coefficient and the rate of heat transfer at the surface.

2. The Lorentz force which appears in the flow-field due to the applied magnetic field reduces the thickness of momentum and concentration boundary layers whereas it has an opposite effect on the thermal boundary layer. The observed effect, however, on the thermal boundary layer is not significant and the Lorentz force marginally increases its thickness. The skin friction increases, whereas the rate of heat transfer at the surface decreases, with increasing strength of magnetic field.

3. The effect of homogeneous and heterogeneous reactions is to decrease the species concentration within the boundary layer region.

4. Heat absorption has the tendency to decrease the thickness of the nanofluid thermal boundary layer whereas it increases the rate of heat transfer at the surface.

Author Contributions: R.N. and P.S. conceived the mathematical model of the problem, B.K.M. designed the computational algorithm and carried out numerical computations, and G.K.M. helped in writing the manuscript. All the authors read and approved the final version of the manuscript.

Conflicts of Interest: All the authors jointly declare that they have no conflict of interest.

\section{References}

1. Choi, S.U.S.; Zhang, Z.G.; Yu, W.; Lockwood, F.E.; Grulke, E.A. Anomalously thermal conductivity enhancement in nanotube suspensions. Appl. Phys. Lett. 2001, 79, 2252-2254.

2. Buongiorno, J.; Hu, W. Nanofluid coolants for advanced nuclear power plants. In Proceedings of the International Congress on Advances in Nuclear Power Plants 2005 (ICAPP05), Seoul, Korea, 15-19 May 2005.

3. Wang, X.Q.; Mazumdar, A.S. Heat transfer characteristics of nanofluids: A review. Int. J. Therm. Sci. 2007, $46,1-19$.

4. Das, S.K.; Choi, S.U.S.; Yu, W.; Pradeep, T. Nanofluids: Science and Technology; Wiley: Hoboken, NY, USA, 2008.

5. Kakac, S.; Pramuanjaroenkij, A. Review of convective heat transfer enhancement with nanofluids. Int. J. Heat Mass Transf. 2009, 52, 3187-3196.

6. Chamkha, A.J.; Rashad, A.M.; Al-Meshaiei, E. Melting effect on unsteady hydromagnetic flow of a nanofluid past a stretching sheet. Int. J. Chem. React. Eng. 2011, 9, A113. 
7. Ibrahim, W.; Shankar, B.; Nandeppanavar, M.M. MHD stagnation poiont flow and heat transfer due to Nanofluid towards a stretching sheet. Int. J. Heat Mass Transf. 2013, 56, 1-9.

8. Vajravelu, K.; Nayfeh, J. Hydromagnetic convection at cone and a wedge. Int. Commun. Heat Mass Transf. 1992, 19, 701-710.

9. Westphal, B.R.; Keiser, D.D.; Rigg, R.H.; Loug, D.V. Production of Metal Waste Forms from Spent Nuclear Fuel Treatment. In Proceedings of the DOE Spent Nuclear Fuel Conference, Salt Lake City, UT, USA, 13-16 December 1994; pp. 288-294.

10. Chamkha, A.J.; Khaled, A.R.A. Similarity solutions for hydromagnetic simultaneous heat and mass transfer by natural convection from an inclined plate with heat generation or absorption. Heat Mass Transf. 2001, 37, 117-123.

11. Kamel, M.H. Unsteady MHD convection through porous medium with combined heat and mass transfer with heat source/sink. Energy Convers. Manag. 2001, 42, 393-405.

12. Chamkha, A.J. Unsteady MHD convective heat and mass transfer past a semi-infinite vertical permeable moving plate with heat absorption. Int. J. Eng. Sci. 2004, 42, 217-230.

13. Alam, M.S.; Rahamn, M.M.; Samad, M.A. Numerical Study of the Combined Free-Forced Convection and Mass Transfer Flow Past a Vertical Porous Plate in a Porous Medium with Heat Generation and Thermal Diffusion. J. Nonlinear Anal. Model. Control 2006, 11, 331-343.

14. Alam, M.S.; Rahman, M.M.; Sattar, M.A. Effects of Chemical Reaction and Thermophoresis on MHD Mixed Convective Heat and Mass Transfer Flow Along an Inclined Plate in the Presence of Heat Generation/Absorption with Viscous Dissipation and Joule Heating. Can. J. Phys. 2008, 86, 1057-1066.

15. Mahatha, B.K.; Nandkeolyar, R.; Das, M.; Sibanda, P. Stagnation point nanofluid flow along a stretching sheet with non-uniform heat generation/absorption and Newtonian heating. Bulg. Chem. Commun. 2017, 49, 977-985.

16. Chaudhary, M.A.; Merkin, J.H. A simple isothermal model for Homogeneous-heterogeneous reactions in boundary layer flow: I. Equal diffusivities. Fluid Dyn. Res. 1995, 16, 311-333.

17. Chaudhary, M.A.; Merkin, J.H. A simple isothermal model for Homogeneous-heterogeneous reactions in boundary layer flow: II. Different Diffusivities for Reactant and Autocatalyst. Fluid Dyn. Res. 1995, 16, 335-359.

18. Merkin, J.H. A model for isothermal Homogeneous-heterogeneous reactions in boundary layer flow. Math. Comput. Model. 1996, 24, 125-136.

19. Chaudhary, M.A.; Merkin, J.H. Homogeneous-Heterogeneous Reactions in Boundary-Layer Flow: Effects of Loss of Reactant. Math. Comput. Model. 1996, 24, 21-28.

20. Khan, W.A.; Pop, I. Flow near the two-dimensional stagnation point on a infinite permeable wall with a Homogeneous-heterogeneous reaction. Commun. Nonliear Sci. Numer. Simul. 2010, 15, 3435-3443.

21. Khan, W.A.; Pop, I. Effect of Homogeneous-heterogeneous reactions on the Visco-elastic fluid towards a stretching sheet. ASME J. Heat Transf. 2012, 134, 064506.

22. Kameswaran, P.K.; Shaw, S.; Sibanda, P.; Murthy, P.V.S.N. Homogeneous-heterogeneous reactions in a nanofluid flow due to a porous stretching sheet. Int. J. Heat Mass Transf. 2013, 57, 465-472.

23. Kameswaran, P.K.; Sibanda, P.; RamReddy, C.; Murthy, P.V.S.N. Dual solutions of stagnation-point flow of a nanofluid over a stretching surface. Bound. Value Probl. 2013, 2013, 1-12.

24. Nandkeolyar, R.; Motsa, S.S.; Sibanda, P. Viscous and Joule Heating in the Stagnation Point Nanofluid Flow Through a Stretching Sheet With Homogenous-Heterogeneous Reactions and Nonlinear Convection. ASME J. Nanotechnol. Eng. Med. 2013, 4, 041002.

25. Cramer, K.; Pai, S. Magnetofluiddynamics for Engineers and Applied Physicists; McGraw Hill Book Company: New York, NY, USA, 1973.

26. Brinkman, H.C. The viscosity of concentrated suspensions and solution. J. Chem. Phys. 1952, 20, 571-581.

27. Aminossadati, S.M.; Ghasemi, B. Natural convection cooling of a localized heat source at the bottom of a nanofluid-filled enclosure. Eur J. Mech. B Fluids 2009, 28, 630-640.

28. Garnett, J.C.M. Colours in metal glasses and in mettaillic films. Philos. Trans. R. Soc. Lond. A 1904, 203, 385-420. 
29. Guérin, C.A.; Mallet, P.; Sentenac, A. Effective-medium theory for finite-size aggregates. J. Opt. Soc. Am. A 2006, 23, 349-358.

30. Motsa, S.S.; Sibanda, P.; Shateyi, S. On a new quasilinearization method for systems of nonlinear boundary value problems. Math. Methods Appl. Sci. 2011, 34, 1406-1413. 